\title{
Condition Help: A Patient- and Family-Initiated Rapid Response System
}

\author{
Elizabeth L. Eden, MD ${ }^{1 \star}$, Laurie L. Rack, DNP, RN², Ling-Wan Chen, $\mathrm{MS}^{3}$, Gregory M. Bump, MD ${ }^{4}$
}

${ }^{1}$ Internal Medicine Residency Program and ${ }^{2}$ Medicine Services, University of Pittsburgh Medical Center, Pittsburgh, Pennsylvania; ${ }^{3}$ Department of Statistics, University of Pittsburgh, Pittsburgh, Pennsylvania; ${ }^{4}$ University of Pittsburgh School of Medicine and University of Pittsburgh Medical Center, Pittsburgh, Pennsylvania.

\begin{abstract}
BACKGROUND: Rapid response teams (RRTs) help in delivering safe, timely care. Typically they are activated by clinicians using specific parameters. Allowing patients and families to activate RRTs is a novel intervention. The University of Pittsburgh Medical Center developed and implemented a patient- and family-initiated rapid response system called Condition Help (CH).

METHODS: When the $\mathrm{CH}$ system is activated, a patient care liaison or an on-duty administrator meets bedside with the unit charge nurse to address the patient's concerns. In this study, we collected demographic data, call reasons, call designations (safety or nonsafety), and outcome information for all $\mathrm{CH}$ calls made during the period January 2012 through June 2015.
\end{abstract}

RESULTS: Two hundred forty patients/family members made $367 \mathrm{CH}$ calls during the study period. Most calls were made by patients $(76.8 \%)$ rather than family members $(21.8 \%)$. Of the 240 patients, $43(18 \%)$ made multiple calls; their calls accounted for $46.3 \%$ of all calls (170/367). Inadequate pain control was the reason for the call in most cases $(48.2 \%)$, followed by dissatisfaction with staff $(12.5 \%)$. The majority of calls involved nonsafety issues (83.4\%) rather than safety issues (11.4\%). In $41.4 \%$ of cases, a change in care was made.

CONCLUSION: Patient- and family-initiated RRTs are designed to engage patients and families in providing safer care. In the $\mathrm{CH}$ system, safety issues are identified, but the majority of calls involve nonsafety issues. Journal of Hospital Medicine 2017;12:157-161. @ 2017 Society of Hospital Medicine
In recent years, rapid response teams (RRTs) have been widely implemented to improve patient safety and quality of care. RRTs traditionally are activated by providers to address a clinically deteriorating patient; trained nurses, respiratory care specialists, and physicians are brought bedside to assist in triage and management. After the Joint Commission ${ }^{1}$ endorsed patient engagement as a strategy for enhancing patient safety, new initiatives were developed to meet the challenge. Programs designed to enhance patient engagement have taken a variety of forms, including educational campaigns encouraging patients to report adverse events, requests for handwashing by providers, and the institution of patient- and family-activated RRTs. ${ }^{2}$ Patient involvement is viewed favorably and has been shown to increase patients' perception of health care quality. ${ }^{3}$ Although these initiatives are presumed helpful in encouraging communication, there is limited evidence that more communication leads to safety improvements. Despite the increasing prevalence of patient-activated RRTs in the United States, they have gone largely unevaluated in the adult population, and their efficacy remains unclear.

\section{CONDITION HELP}

Condition Help $(\mathrm{CH})$ is a patient- and family-initiated RRT designed to prevent medical errors and communication

\footnotetext{
*Address for correspondence and reprint requests: Elizabeth L. Eden, MD, Internal Medicine Residency Program, 7th Floor, Montefiore Hospital, 200 Lothrop St, Pittsburgh PA 15213; Telephone: 412-802-6648; Fax: 412-692-4499; E-mail: edenel@upmc.edu

Received: June 9, 2016; Revised: September 8, 2016; Accepted: September 18, 2016

2017 Society of Hospital Medicine DOI 10.12788/jhm.2697
}

problems and improve patient safety. Patients and families are encouraged to call the $\mathrm{CH}$ hotline if they believe that there has been a breakdown in care or that their health is in imminent danger. This RRT was inspired by the case of Josie King, an 18-month-old girl who died of preventable causes at a large children's hospital. ${ }^{4}$ After her daughter's death, Sorrel King started the Josie King Foundation, an organization committed to preventing medical errors and creating a culture of patient safety. With the support of this foundation, CH was launched in 2005 at the Children's Hospital of Pittsburgh at the University of Pittsburgh Medical Center (UPMC). Later it was implemented at the UPMC adult tertiary-care center, and now it is available in all UPMC facilities.

On admission, patients receive a brochure that details the purpose of $\mathrm{CH}$ and provides examples of when and how to call the $\mathrm{CH}$ hotline. In this brochure, patients are instructed to call $\mathrm{CH}$ in 3 situations: "1) There is an emergency and you cannot get the attention of hospital staff, 2) You see a change in the patient's condition and the health care team is not recognizing the concern, or 3) There is breakdown in how care is given or uncertainty over what needs to be done." These instructions are printed on bulletins placed in elevators and hallways throughout the hospital. Patients and families may activate the system at any time and can even do so from home.

When a patient or family member calls the hotline, an operator notifies the $\mathrm{CH}$ team. This team, which consists of a patient care liaison (or an on-duty administrator) and the unit charge nurse, convenes bedside to address the patient's concern. The team was designed without a physician to ensure that the primary team remains in charge of the 
care plan. $\mathrm{CH}$ is kept separate from our traditional RRT and does not compete for resources (personnel, equipment, time) with the RRT, which is designed to address a clinically deteriorating patient.

In this article, we describe the characteristics of patients for whom $\mathrm{CH}$ was activated at our adult hospital. We also describe reasons for calls, whether changes in care were implemented, and outcomes, including traditional RRT activation, transfer to intensive care unit (ICU), and inpatient mortality. As $\mathrm{CH}$ was designed with patient safety as a goal, we tracked 2 types of calls, those involving safety issues and those involving nonsafety issues.

\section{METHODS}

This study was approved by the quality improvement committee at the University of Pittsburgh and was considered exempt from review by the university's Institutional Review Board.

Our integrated health system consists of more than 20 hospitals serving a tristate region. UPMC Presbyterian and UPMC Montefiore are adult tertiary-care referral hospitals with more than 750 medical/surgical beds and 150 critical care beds and more than 30,000 annual inpatient admissions. These hospitals are physically connected and function as a single large medical center. We reviewed all $\mathrm{CH}$ events that occurred at this combined hospital during the period January 2012 through June 2015. The dates coincided with $\mathrm{CH}$ data acquisition.

$\mathrm{CH}$ was available 24 hours a day 7 days a week. A patient care liaison (or an on-duty administrator) and the unit charge nurse responded to $\mathrm{CH}$ calls. Data from all calls included date and time of call, day of week, primary service, patient location, unique patient identifiers, call initiator (patient or family), whether a call led to changes in care, and primary reason for call. Each call reason was sorted into 1 of 10 categories: pain control, staff problem, lack of communication between patient/family and care team, questions about patient management, care delays, delays in a particular service, questions about discharge, administrative issues, acute psychiatric needs, and unknown/other. In addition, after a call, we reviewed all charts to determine if a safety issue was involved; Dr. Eden and Dr. Bump independently reviewed calls for safety issues and discussed any differences until they reached consensus. We also recorded outcomes, including activation of a traditional RRT or transfer to ICU within 24 hours of $\mathrm{CH}$ call, inpatient mortality, and against medical advice (AMA) discharges. Given that many calls were made by patients who called more than once (during a single admission or over multiple admissions), we also sorted patients into one-time callers and repeat callers for comparison. Patient satisfaction data were unavailable for review.

Patient demographic data are presented as means, standard deviations, and percentages, and call characteristics as percentages. Chi-square tests and $t$ tests were used for analyses except for comparisons having few observations. For those, Fisher exact test was used. All analyses were performed with SAS Version 9.4 (SAS Institute, Cary, North Carolina).

\section{RESULTS}

From January 2012 through June 2015, 367 CH calls were made, about 105 annually. During this period, there were about 33,000 admissions, 800 combined grievances and complaints, 170 AMA discharges, 155 cardiac arrests, 2300 traditional RRT activations, and 1200 inpatient deaths per year. The $367 \mathrm{CH}$ calls were made by 240 patients (Table 1). Of these 240 patients, 43 (18\%) activated the $\mathrm{CH}$ team with multiple calls; their calls accounted for $(46.3 \%)$ of all calls (170/367). The majority of calls were made by patients $(76.8 \%)$ rather than family members (21.8\%). Mean (SD) patient age was 45.8 (17.4) years. Mean (SD) number of admissions per patient per year was 2.7 (3.5). More events were activated for patients admitted to medical services (66\%) than surgical services (34\%). Calls were evenly distributed between time of day and day of week.

The most common reason for $\mathrm{CH}$ calls was inadequate pain control $(48.2 \%)$, followed by dissatisfaction with staff $(12.5 \%)$; the remaining calls were evenly distributed among the other categories. The majority of calls involved nonsafety issues $(83.4 \%$ ) rather than safety issues $(11.4 \%)$; in $5.2 \%$ of calls, the distinction could not be made because of lack of information (Table 2). In 152 (41.4\%) of the 367 total calls, a change in care or alteration in management was made. Of these 152 calls, 99 (65.1\%) involved distinct changes in the care plan, such as medication changes, imaging or additional testing, or consultation with other physicians; the other 53 calls (34.9\%) involved additional patient counseling or nonmedical changes. Our traditional RRT was activated within 24 hours of $\mathrm{CH}$ in 19 cases (5.2\%); of the 19 patients, 6 were transferred to ICU. Seven patients $(2.9 \%)$ died during admission. Twelve (3.3\%) were discharged AMA. We compared outcomes of patients who made safety-issue calls with those of patients who made nonsafety-issue calls. The composite outcome of RRT activation, ICU transfer, and mortality was found for $6(14.3 \%)$ of the 42 safety-issue calls and 15 (4.9\%) of the 306 nonsafety-issue calls $(P=0.0291)$.

The unexpected high rate of repeat calling prompted us to compare the characteristics of one-time and repeat callers. Repeat callers were younger: Mean age was 39.3 (12.8) years for repeat callers and 47.2 (17.9) years for one-time callers $(P=0.0012)$. Repeat callers had more admissions per year: Mean (SD) number of admissions was 5.67 (5.4) for repeat callers and $2.09(2.5)$ for one-time callers $(P=0.0001)$. One-time and repeat callers did not differ with respect to race or sex. Compared with one-time callers, repeat callers were more often $(P=0.002)$ admitted to medical services (74.7\%) than surgical services $(58.9 \%)$. For repeat callers, a larger percentage of calls $(P<0.0001)$ were made by patients $(93.5 \%)$ rather than families (62.4\%). Calls about pain were more often $(P<0.0001)$ made by repeat callers $(62.3 \%)$ than one-time callers (36\%), calls involving safety issues were less often $(P<0.0001)$ made by repeat callers $(5.9 \%)$ than onetime callers $(16.2 \%)$, and changes in care were made less often $(P<0.0001)$ for repeat callers $(32.9 \%)$ than one-time callers $(48.7 \%)$. Between-group differences in rates of RRT 
TABLE 1. Descriptors and Outcomes of Patients Who Called Condition Help

\begin{tabular}{|c|c|c|c|c|}
\hline Descriptor/Outcome & \multicolumn{3}{|c|}{ Callers } & $P^{a}$ \\
\hline Total calls, $n(\%)$ & 367 & $197(53.7)$ & $170(46.3)$ & - \\
\hline Male & $91(38)$ & $79(40)$ & $12(28)$ & \\
\hline Female & $149(62)$ & $118(60)$ & $31(72)$ & \\
\hline Race, n (\%) & & & & $0.4275^{b}$ \\
\hline Other & $8(3.3)$ & $8(4)$ & 0 & \\
\hline Mean (SD) age, y & $45.8(17.4)$ & $47.2(17.9)$ & $39.3(12.8)$ & $0.0012^{c}$ \\
\hline Mean (SD) admissions/y & $2.72(3.5)$ & $2.09(2.5)$ & $5.67(5.4)$ & $0.0001^{c}$ \\
\hline Callers, n (\%) & & & & $<0.0001^{b}$ \\
\hline Patient & $282(76.8)$ & $123(62.4)$ & $159(93.5)$ & \\
\hline Family & $80(21.8)$ & $69(35)$ & $11(6.5)$ & \\
\hline Daytime & $183(49.9)$ & $88(44.7)$ & 95 (55.9) & $0.032^{d}$ \\
\hline Night & $184(50.1)$ & $109(55.3)$ & $75(44.1)$ & \\
\hline Admitting service, $n$ (\%) & & & & $0.002^{d}$ \\
\hline Medicine & $243(66.2)$ & $116(58.9)$ & $127(74.7)$ & \\
\hline Surgery & $124(33.8)$ & $81(41.1)$ & $43(25.3)$ & \\
\hline \multicolumn{5}{|l|}{ Reason for call, $\mathrm{n}(\%)$} \\
\hline Pain & 177 (48.2) & $71(36)$ & $106(62.4)$ & $<0.0001^{\mathrm{d}}$ \\
\hline Staff & $46(12.5)$ & $26(13.2)$ & $20(11.8)$ & \\
\hline Communication & $22(6)$ & $16(8.1)$ & $6(3.5)$ & \\
\hline Management & $26(7.1)$ & $17(8.6)$ & $9(5.3)$ & \\
\hline Discharge & $26(7.1)$ & $16(8.1)$ & $10(5.9)$ & \\
\hline Timing or delays & $16(4.4)$ & $12(6.1)$ & $4(2.4)$ & \\
\hline Safety & $42(11.4)$ & $32(16.2)$ & $10(5.9)$ & \\
\hline Nonsafety & $306(83.4)$ & $148(75.1)$ & $158(92.9)$ & \\
\hline Unknown & $19(5.2)$ & $17(8.6)$ & $2(1.2)$ & \\
\hline Change made, $\mathrm{n}(\%)$ & & & & $<0.0001^{b}$ \\
\hline Yes & $152(41.4)$ & $96(48.7)$ & $56(32.9)$ & \\
\hline No & $188(51.2)$ & $77(39.1)$ & $111(65.3)$ & \\
\hline Unknown & $27(7.4)$ & $24(12.2)$ & $3(1.8)$ & \\
\hline Traditional rapid response call, $n$ (\%) & & & & $0.8150^{b}$ \\
\hline Yes & $19(5.2)$ & $11(5.6)$ & $8(4.7)$ & \\
\hline No & $348(94.8)$ & $186(94.4)$ & $162(95.3)$ & \\
\hline Escalation to ICU, n (\%) & & & & $0.6901^{b}$ \\
\hline Yes & $6(1.6)$ & $4(2)$ & $2(1.2)$ & \\
\hline No & $361(98.4)$ & $193(98)$ & $168(98.8)$ & \\
\hline Alive at discharge, $n(\%)$ & & & & $0.3573^{b}$ \\
\hline Yes & $233(97.1)$ & $190(96.4)$ & $43(100)$ & \\
\hline No & $7(2.9)$ & $7(3.6)$ & $0(0)$ & \\
\hline AMA discharge, $n(\%)$ & & & & $1.0000^{\mathrm{b}}$ \\
\hline Yes & $12(3.3)$ & $6(3)$ & $6(3.5)$ & \\
\hline No & $355(96.7)$ & $191(97)$ & $164(96.5)$ & \\
\hline $\begin{array}{l}\text { aP values for differences between one-time } \\
\text { "By Fisher exact test because of small sampl } \\
\text { 'By } 2 \text {-sided } t \text { test with unequal variance. } \\
\text { 'By } \chi^{2} \text { test. } \\
\text { NOTE: Abbreviations: AMA, against medical a }\end{array}$ & & & & \\
\hline
\end{tabular}


TABLE 2. Examples of Condition Help Calls Attributed to Safety and Nonsafety Issues

\begin{tabular}{|c|c|c|}
\hline \multicolumn{3}{|l|}{ Call } \\
\hline Designation & Scenario & Result \\
\hline Safety & $\begin{array}{l}\text { Patient discharged by team felt poorly, developed fever, and called } \mathrm{CH} \text { to contest } \\
\text { discharge. }\end{array}$ & $\begin{array}{l}\text { Discharge canceled. Caller remained inpatient for infection work-up and } \\
\text { treatment. }\end{array}$ \\
\hline Safety & $\begin{array}{l}\text { Patient with ventriculoperitoneal shunt was admitted for a fall. While inpatient, fell again } \\
\text { and developed headache, prompting } \mathrm{CH} \text { call. }\end{array}$ & Patient underwent head imaging and shunt evaluation. \\
\hline Safety & $\begin{array}{l}\text { Family member of critically ill ventilated patient called } \mathrm{CH} \text { about communication issues. } \\
\text { Had received contradictory plans from different providers and requested clarification. }\end{array}$ & Teams and family met to discuss care plan. \\
\hline Safety & $\begin{array}{l}\text { Patient called } \mathrm{CH} \text { to report mishandling of PICC by nurse. Noted that nurse did not use } \\
\text { sterile technique during PICC maintenance and did not aspirate after administering } \\
\text { alteplase to declog. }\end{array}$ & $\begin{array}{l}\text { Case was discussed with charge nurse, who provided nursing education and } \\
\text { changed nursing assignment. }\end{array}$ \\
\hline Safety & $\begin{array}{l}\text { Patient was admitted for tibial fracture and underwent surgery. Called } \mathrm{CH} \text { for uncontrolled } \\
\text { pain after procedure. }\end{array}$ & $\begin{array}{l}\text { Surgeon was called to patient's bed to assess for compartment syndrome. Pain } \\
\text { medication was increased. }\end{array}$ \\
\hline Nonsafety & Patient called $\mathrm{CH}$ to request change in diet from consistent-carbohydrate to regular. & Diet was changed. \\
\hline Nonsafety & $\begin{array}{l}\text { Patient with chronic abdominal pain and known drug-seeking behavior called } \mathrm{CH} \text { to } \\
\text { request increase in pain medication. }\end{array}$ & $\begin{array}{l}\text { Primary physician discussed issue with patient and established care plan. Pain } \\
\text { medication was not increased. }\end{array}$ \\
\hline Nonsafety & Patient upset about waiting 2 days for MRI. & Team unable to expedite routine MRI. \\
\hline Nonsafety & Patient called $\mathrm{CH}$ because was concerned that parasites were eating her skin. & Psychiatry was consulted for management. \\
\hline Nonsafety & $\begin{array}{l}\text { Family member called } \mathrm{CH} \text { when patient was transferred from ICU to step-down unit. } \\
\text { Family was worried patient would receive inferior care, and wanted her to remain in ICU. }\end{array}$ & $\begin{array}{l}\text { Family member was educated about step-down unit staffing and was assured } \\
\text { that transfer was appropriate. }\end{array}$ \\
\hline
\end{tabular}

activation, transfer to ICU, inpatient mortality, and AMA discharges were not significant.

\section{DISCUSSION}

Patient- and family-activated RRTs provide unique opportunities for patient and family engagement during inpatient hospital stays. Our study described the results obtained with use of a well-established patient-activated RRT over several years, one of the longer observation periods reported in the literature. We found that, with use of patient-activated RRTs, patient safety issues were identified, though these were far outnumbered by nonsafety issues.

Almost half of all $\mathrm{CH}$ events were related to pain. Pain as the primary driver for RRT activation may be attributable to several factors, including degree of illness, poor communication about pain management expectations, positive reinforcement of narcotic-seeking behavior as a result of $\mathrm{CH}$ activation, and high rate of opiate use in the catchment area. A striking finding of our analysis was repeat calling; only 43 $(18 \%)$ of the 240 callers were repeat callers, but they made almost half of all the calls. In some cases, during a single admission, multiple calls were made because the first had no effect on care or management; more typically, though, multiple calls were made over several admissions. Repeat callers were admitted more often per year, and they used hospital services more. They should be further studied with a goal of designing programs that better meet their needs and that prospectively address expectations of pain control.

Our study was unique in describing several outcomes related to $\mathrm{CH}$ events. We found that traditional RRTs were seldom activated, level of care was seldom escalated, and mortality was rare, though these outcomes occurred more often for safety-issue calls than nonsafety-issue calls. We also found that activation of $\mathrm{CH}$ teams often led to changes in medical management, though we could not determine whether these changes in care led to different patient outcomes.

Patient-initiated RRTs are described in a limited number of pediatric and adult studies, all with findings differing from ours. In the pediatric models, most calls were initiated by family members, were less frequent, and tended to signal higher patient acuity. ${ }^{5,6}$ For example, in a pediatric RRT model, ${ }^{5}$ family members activated the RRT only twice within the study year, but both calls resulted in ICU transfer. Most descriptions of patient-activated RRTs in adult hospitals are from pilot studies, which similarly identified infrequent RRT calls but often did not identify call reasons or specific outcomes. ${ }^{7}$ A single-center study concluded that, after implementation of a mixed-model RRT ${ }^{8}$ - a traditional practitioner-activated RRT later enhanced with a patient/family activation mechanism-non-ICU codes decreased, and there was a statistically significant drop in hospital-wide mortality rates. However, this RRT was patient-activated only 25 times over 2 years, and the specific outcomes of those events were not described.

Other initiatives have been designed to enhance patient care and communication. Purposeful rounding systems ${ }^{9}$ involve hourly rounding by bedside nurses and daily rounding by nurse leaders to improve timely patient care and provide proactive service. Such systems ideally preempt calls involving dissatisfaction and nonsafety issues. Although they would reduce the number of patient-dissatisfaction calls made in the $\mathrm{CH}$ system, they may not be any better than the $\mathrm{CH}$ system is in its main purpose, identifying safety issues. In addition, whether patient-activated RRTs or purposeful rounding systems are better at addressing patient dissatisfaction is unclear. 
This study had its limitations. First, like other studies, it was a single-center observational study without a concurrent control group. Second, because $\mathrm{CH}$ was first implemented 10 years ago, we could not compare patient outcomes or traditional RRT use before and after program initiation. Third, our study cohort consisted of patients hospitalized at one academic tertiary-care center in one region, and the hospital is a training site for multiple residencies and fellowships. These factors likely affect the generalizability of our data to smaller or community-based centers. Fourth, some determinations were subjective (eg, whether calls involved safety or nonsafety issues). We tried to minimize bias by having 2 authors independently review cases, but the process did not reflect patient experience or perspective. Fifth, our hospital adopted its traditional RRT years before its $\mathrm{CH}$ system. The criteria used by hospital personnel for traditional RRT activation are designed to encourage staff to call for help at early signs of patient deterioration. Consequently, traditional RRT activations substantially outnumber $\mathrm{CH}$ calls. Whether this resulted in fewer $\mathrm{CH}$ safety calls is unclear. Sixth, we did not capture the financial implications of using $\mathrm{CH}$ teams.

Although patient-activated RRTs identified patient safety issues, questions about the utility or necessity of these RRTs remain. In our era of limited hospital resources, the case has not been definitively made that these teams are practical, based on patient outcomes, though other studies have found improved patient satisfaction. ${ }^{7}$ Most of the RRT calls in our study involved patient dissatisfaction and communication issues. $\mathrm{CH}$ may not be the ideal approach for managing these issues, but it represents the last line of patient advocacy once other systems have failed.

We think patient-activated RRTs have the potential to effect patient engagement in safe care. Given the importance of establishing a culture of patient safety and engagement, and increased detection of safety-related events, $\mathrm{CH}$ remains active throughout our hospital system. Newer iterations of $\mathrm{CH}$ may benefit from stricter language in defining appropriate occasions for calling RRTs, and from descriptions of other resources for patient advocacy within the hospital. These modifications could end up restricting RRT activations to patient complaints and preserving $\mathrm{CH}$ resources for patients with safety concerns. Our study lays the groundwork for oth- er institutions that are considering similar interventions. Studies should now start evaluating how well patient- and family-activated RRTs improve patient satisfaction, staff satisfaction, and patient outcomes.

\section{CONCLUSION}

Patient-and family-activated RRTs were designed to engage patients and families in safe care. Although $\mathrm{CH}$ detects patient safety issues, these are far outnumbered by nonsafety issues. $\mathrm{CH}$ demonstrates a commitment to patient engagement and a culture that emphasizes patient safety.

\section{Acknowledgments}

This work was presented as a poster at the annual meeting of the Society of Hospital Medicine; March 6-9, 2016; San Diego, CA.

Disclosure: Nothing to report.

\section{References}

1. Joint Commission. Improving America's Hospitals: The Joint Commission's Annual Report on Quality and Safety 2008. http://www.jointcommission.org/assets/1/6/2008_Annual_Report.pdf. Published November 2008. Accessed May 4, 2016.

2. Berger Z, Flickinger TE, Pfoh E, Martinez KA, Dy SM. Promoting engagement by patients and families to reduce adverse events in acute care settings: a systematic review. BMJ Qual Saf. 2014;23(7):548-555.

3. Weingart SN, Zhu J, Chiappetta L, et al. Hospitalized patients' participation and its impact on quality of care and patient safety. Int J Qual Health Care. 2011;23(3):269-277

4. Kennedy P, Pronovost P. Shepherding change: how the market, healthcare providers, and public policy can deliver quality care for the 21st century. Crit Care Med. 2006;34(3 suppl):S1-S6

5. Ray EM, Smith R, Massie S, et al. Family alert: implementing direct family activation of a pediatric response team. Jt Comm J Qual Patient Saf. 2009;35(11): 575-580.

6. Dean BS, Decker MJ, Hupp D, Urbach AH, Lewis E, Benes-Stickle J. Condition Help: a pediatric rapid response team triggered by patients and parents. J Healthc Qual. 2008;30(3):28-31.

7. Vorwerk J, King L. Consumer participation in early detection of the deteriorating patient and call activation to rapid response systems: a literature review. J Clin Nurs. 2015;25(1-2):38-52.

8. Gerdik C, Vallish RO, Miles K, Godwin SA, Wludyka PS, Panni MK. Successful implementation of a family and patient activated rapid response team in an adult level 1 trauma center. Resuscitation. 2010;81(12):1676-1681

9. Hancock KK. From the bedside: purposeful rounding essential to patient experience. Association for Patient Experience website. http://www.patientexperience.org/Resources/Newsletter/Newsletters/Articles/2014/From-the-Bedside-Purposeful-Rounding-Essential-to.aspx. Published February 27, 2014. Accessed July 25, 2016. 